\title{
Erratum: Berry curvature and orbital angular momentum of electrons in angle-resolved photoemission spectroscopy [Phys. Rev. B 91, 245133 (2015)]
}

\author{
Ryuji Takahashi and Naoto Nagaosa \\ (Received 14 December 2016; published 23 February 2017)
}

DOI: 10.1103/PhysRevB.95.079905

There are mistakes in our proposal for experimental setup in Sec. IV of our original paper; the electric field is treated as imaginary by error in Eq. (23). In the right-hand side of the first equation in Eq. (23), the operator between $|\mathbf{k}\rangle$ and $\left|\mathbf{k}^{\prime}\right\rangle$ is the time-evolution operator in the interaction representation. Then, by the relation $\left\langle\mathbf{k}|X| \mathbf{k}^{\prime}\right\rangle \sim-i\left\langle\mathbf{k}\left|\partial_{k_{x}}\right| \mathbf{k}^{\prime}\right\rangle$, the second term in the second equation in Eq. (23) should be changed to $b \rightarrow i b$, and consequently, the observation scheme in Sec. IV breaks down.

We give another scheme for observing the Hall current of the electron with orbital angular momenta (OAM) and claim that the observation of the OAM is experimentally feasible. We consider a situation where the photoelectron passes through a plate which absorbs injected electrons. We put a plate parallel to the surface (the $x y$ plane) of the material where photoelectron comes from. The plate is not magnetically ordered. When we focus only on the electrons passing through the plate, the Hamiltonian is effectively given as $H=H_{0}-i \frac{\gamma p_{z}}{2 m_{e}}$, where $H_{0}$ is the Hamiltonian of free electrons, $m_{e}$ is the electron mass, and $\gamma^{-1}$ is the attenuation length determined by kind of chemical compounds of the plate. At $t=0$ the electron enters the region of the plate with finite thickness, and then the wave function is written as

$$
\Psi^{\prime}\left(\mathbf{R}, \mathbf{k}_{c}, t\right)=e^{-i t H_{0}-\frac{\gamma p_{z}}{2 m_{e}} t} \Psi\left(\mathbf{R}, \mathbf{k}_{c}\right)
$$

Here, we assume that the attenuation length has the form $\gamma=\gamma_{0}\left[1+\epsilon_{s}\left(X-X_{g}\right)\right]$ where $\epsilon_{s}$ is the modulation of the attenuation length and $X_{g}$ is the center of the modulation. Then, when the modulation and transit time $(t)$ are small, the wavefunction has the form

$$
\Psi^{\prime}\left(\mathbf{R}, \mathbf{k}_{c}, t\right)=e^{-i t H_{0}-\frac{\gamma_{0} p_{z}}{2 m_{e}} t}\left[1-\frac{\gamma_{0} \epsilon_{s} p_{z}}{2 m_{e}} t\left(X-X_{g}\right)\right] \Psi\left(\mathbf{R}, \mathbf{k}_{c}\right),
$$

and we have the expectation value

$$
\begin{aligned}
\left\langle P_{y}^{\prime}\right\rangle & =\frac{\left\langle\Psi^{\prime}\left(\mathbf{R}, \mathbf{k}_{c}, t\right)\left|k_{y}\right| \Psi^{\prime}\left(\mathbf{R}, \mathbf{k}_{c}, t\right)\right\rangle}{\left\langle\Psi^{\prime}\left(\mathbf{R}, \mathbf{k}_{c}, t\right) \mid \Psi^{\prime}\left(\mathbf{R}, \mathbf{k}_{c}, t\right)\right\rangle}=\frac{\left\langle k_{y}\right\rangle-\frac{\gamma_{0} \epsilon_{s} p_{z}}{m_{e}} t\left\langle k_{y}\left(X-X_{g}\right)\right\rangle}{1-\frac{\gamma_{0} \epsilon_{s} p_{z}}{m_{e}} t\left\langle\left(X-X_{g}\right)\right\rangle} \\
& =\left\langle k_{y}\right\rangle-\frac{\gamma_{0} \epsilon_{s} p_{z}}{m_{e}} t\left[\left\langle k_{y}\left(X-X_{g}\right)\right\rangle-\left\langle k_{y}\right\rangle\left\langle\left(X-X_{g}\right)\right\rangle\right],
\end{aligned}
$$

where $\langle\mathcal{O}\rangle=\left\langle\Psi\left(\mathbf{R}, \mathbf{k}_{c}\right)|\mathcal{O}| \Psi\left(\mathbf{R}, \mathbf{k}_{c}\right)\right\rangle$ and $\left\langle\mathcal{O}^{\prime}\right\rangle=\frac{\left\langle\Psi^{\prime}\left(\mathbf{R}, \mathbf{k}_{c}, t\right)|\mathcal{O}| \Psi^{\prime}\left(\mathbf{R}, \mathbf{k}_{c}, t\right)\right\rangle}{\left\langle\Psi^{\prime}\left(\mathbf{R}, \mathbf{k}_{c}, t\right) \mid \Psi^{\prime}\left(\mathbf{R}, \mathbf{k}_{c}, t\right)\right\rangle}$, assuming the condition $\frac{\gamma_{0} \epsilon_{s} p_{z}}{m_{e}} t\langle X\rangle \sim-\frac{\gamma_{0} \epsilon_{s} p_{z}}{m_{e} \sigma} t \ll 1$ for $X_{g}=0 . \sigma$ corresponds to the size of the wave packet in momentum space (see original paper). The momentum shift becomes

$$
\left\langle P_{y}^{\prime}\right\rangle=\left\langle k_{y}\right\rangle-\frac{\gamma_{0} \epsilon_{s} p_{z}}{m_{e}} t\left(\left\langle k_{y} X\right\rangle-\left\langle k_{y}\right\rangle\langle X\rangle\right)
$$

In the same manner we obtain the change of the momentum $\left\langle P_{x}^{\prime}\right\rangle$ by the anisotropy of $\gamma_{0}$ along the $y$ axis, $\gamma=\gamma_{0}\left(1+\epsilon_{s} Y\right)$, and we have

$$
\left\langle L_{z}\right\rangle=\frac{m_{e}}{\gamma_{0} \epsilon_{s} p_{z} t}\left(\left\langle\delta P_{x}\right\rangle-\left\langle\delta P_{y}\right\rangle\right),
$$

where $\left\langle\delta P_{i}\right\rangle=\left\langle P_{x}^{\prime}\right\rangle-\left\langle P_{i}\right\rangle$. Here, we choose $\frac{\gamma_{0} p_{z}}{m_{e}} t \sim 1$, and then the condition $\epsilon_{s}<\sigma$ is obtained. The thickness of the plate is given by $\gamma^{-1}$; typically $\gamma^{-1}$ is given by the order of the inelastic scattering length $\sim 0.1-10 \mathrm{~nm}$ [1]. The momentum shift $Q$ is estimated as

$$
\frac{Q}{\hbar} \sim \frac{\gamma_{0} \epsilon_{s} p_{z}}{\hbar m_{e}} t\left\langle L_{z}\right\rangle \sim \epsilon_{s} \frac{1}{\hbar}\left\langle L_{z}\right\rangle \sim \epsilon_{s} \frac{\hbar^{2} v^{2} \sigma^{2}}{\Delta^{2}}<k_{0},
$$

with $k_{0} \equiv \frac{\hbar^{2} v^{2} \sigma^{2}}{\Delta^{2}}=7.07 \times 10^{5} \mathrm{~m}^{-1}$, where the same values in the previous paper are used for $v, \sigma$, and $\Delta$. Experimentally, $\epsilon_{s}$ can be obtained by methods such as strain or junction. Here, we consider a junction of materials by connecting two materials labeled by $\mathrm{A}$ and $\mathrm{B}$, which have the attenuation lengths $\gamma_{A}^{-1}$ and $\gamma_{B}^{-1}$, respectively. The middle of the junction region is composed of the alloy of $\mathrm{A}$ and $\mathrm{B}$, and $\gamma$ can be assumed to be proportional to the density of the two materials. According to scattering theory, the intensity after passing through the target is given as $I \propto \mathrm{e}^{-n \ell \Sigma}$, where $n$ is the density of the target, $\ell$ is the length of the target region, and $\Sigma$ is the total scattering cross section determined by component of the target; in the present case $\gamma$ corresponds to $n \Sigma$. 
Then, for example $\gamma_{A} \ll \gamma_{B}$, we have $\epsilon_{S}=\frac{2}{L} \frac{\gamma_{B}-\gamma_{A}}{\gamma_{A}+\gamma_{B}} \sim 2 L^{-1}$, where $L$ is the width of the junction region, and when $L \sim \sigma^{-1}$, we have the largest value of the Hall velocity $\sim \frac{\hbar k_{0}}{m_{e}}$ obtained by the above relation.

[1] I. Lindau and W. E. Spicer, J. Electron Spectrosc. Relat. Phenom. 3, 409 (1974). 